\title{
Frontiers in clinical andrology
}

\author{
Ashok Agarwal $^{1}$, Chak-Lam $\mathrm{Cho}^{2}$, Ahmad Majzoub ${ }^{3}$, Sandro C. Esteves ${ }^{4,5,6}$ \\ ${ }^{1}$ American Center for Reproductive Medicine, Cleveland Clinic, Cleveland, OH, USA; ${ }^{2}$ Division of Urology, Department of Surgery, Kwong Wah \\ Hospital, Hong Kong; ${ }^{3}$ Department of Urology, Hamad Medical Corporation, Doha, Qatar; ${ }^{4}$ ANDROFERT, Andrology and Human Reproduction \\ Clinic, Referral Center for Male Reproduction, Campinas, SP, Brazil; ${ }^{5}$ Division of Urology, University of Campinas (UNICAMP), Campinas, SP, \\ Brazil; ${ }^{6}$ Department of Clinical Medicine, Faculty of Health, Aarhus University, Aarhus, Denmark \\ Correspondence to: Ashok Agarwal. Professor and Director, American Center for Reproductive Medicine, Cleveland Clinic, Mail Code X-11, 10681 \\ Carnegie Avenue, Cleveland, OH 44195, USA. Email: AGARWAA@ccf.org.
}

Submitted Sep 12, 2017. Accepted for publication Sep 12, 2017.

doi: 10.21037/tau.2017.09.07

View this article at: http://dx.doi.org/10.21037/tau.2017.09.07

Since the introduction of intracytoplasmic sperm injection (ICSI) in 1992 (1) the research into the clinical management of infertile men slowed to a grind in the last three decades (2). Attention has been drawn to assisted reproductive technology (ART) in improving embryo quality and pregnancy outcome. The enthusiasm in sperm function tests, including post coital and sperm penetration tests, in 1990s disappeared and none of these tests have been widely studied clinically. Despite all the pitfalls, conventional semen analysis has remained as one-and-only cornerstone in the evaluation of infertile men over the years (3). The increasing use of ICSI in bypassing severe male factors renders semen analysis a screening tool merely for the presence or absence of sperm. Despite its widespread use, the live birth rate utilizing ICSI as the treatment of male factor infertility is around $30 \%$ only (4). This clearly illustrates the pivotal role of paternal contribution to fertilization and development of healthy offspring. A comprehensive evaluation of infertile men and correction of male factors represent the way to improve both natural pregnancy and ART outcomes.

The recognition of sperm quality in addition to semen parameters started in 1950s (5). But it is not until 1970s that the implication of sperm DNA damage on fertility is identified (6). Following the introduction of various sperm DNA fragmentation (SDF) assays in 1980s and 1990s, the pace of research in this area accelerated. Thanks to the efforts of numerous investigators in the field, extensive studies have revealed the association between SDF and reproductive outcomes and the etiology of SDF is being increasingly understood (7). More recently, the emerging evidence on the treatment strategies of high SDF substantiate SDF test as a useful tool in the evaluation of infertile men (7).

While SDF testing is increasingly becoming available in specialized andrology laboratories, its clinical indication remains poorly defined. The practice recommendations by Agarwal et al. represent the first evidence-based attempt to identify the circumstances in which SDF testing should be of the greatest clinical value (8). The review has received feedbacks from experts in the field and published as expert commentaries in this Special Issue of Translational Andrology and Urology. Each of the commentary provides an insight into the topic of SDF from a different perspective. In our quest for generating a wealth of scientific information, the guest editors have responded carefully to the key controversies and opinions highlighted in each expert commentary article. The guest editors have used an evidence-based approach in their writing method. The commentary-response format aims at providing a comprehensive unbiased overview on the subject and illustrates the important principles of SDF from every possible angle. The Special Issue also includes several important articles that summarize the most recent advances of SDF testing. The current utilization of SDF tests was studied by Majzoub et al. using a questionnaire based survey from fertility specialists globally (9). The topic of clinical utility of SDF testing was also studied in the format of strength-weakness-opportunities-threat (SWOT) analysis by Esteves et al. in another chapter (10). A concise practice recommendation was included as a quick reference for clinicians (11). Finally, the latest 
evidence on the role of SDF in male infertility was further refined in a clinical practice guidelines of the Society for Translational Medicine (12).

Currently, the routine use of SDF tests in male factor evaluation is generally not supported by professional societies (13). However, the potential role of SDF has been acknowledged in the latest American Society for Reproductive Medicine (ASRM), American Urological Association (AUA) and European Association of Urology (EAU) guidelines on male infertility (13-15). We believe that a single test with clear cut-off values for fertility assessment is probably not available in view of the dynamic interaction among multiple factors in the complex reproductive system. The uniqueness of SDF testing in providing assessment of genetic content of the male gamete should be considered as complementary to conventional semen analysis in improving male factor evaluation. The evolving evidence on SDF testing in infertile men should be reviewed timely. We believe that the "benchto-bedside" approach in translational medicine (16) will transform the advancements in SDF testing to the clinical benefit of infertile couples. This Special Issue serves as a firm foundation by compiling the achievements of researchers and specialists around the world in shedding light on clinical application of SDF tests. A revolution in the field of male infertility which may drastically change clinical management can be anticipated in the coming years. The wider clinical application of SDF testing will represent a major force in the forward movement in the field with the continuing efforts by all the frontiers in clinical andrology.

\section{Acknowledgements}

None.

\section{Footnote}

Conflicts of Interest: The authors have no conflicts of interest to declare.

\section{References}

1. Palermo G, Joris H, Devroey P, et al. Pregnancies after intracytoplasmic injection of single spermatozoon into an oocyte. Lancet 1992;340:17-8.

2. Schlegel PN, Girardi SK. Clinical review 87: In vitro fertilization for male factor infertility. J Clin Endocrinol
Metab 1997;82:709-16.

3. Esteves SC. Clinical relevance of routine semen analysis and controversies surrounding the 2010 World Health Organization criteria for semen examination. Int Braz J Urol 2014;40:443-53.

4. Neri QV, Tanaka N, Wang A, et al. Intracytoplasmic sperm injection. Accomplishments and qualms. Minerva Ginecol 2004;56:189-96.

5. Leuchtenberger C, Schrader F, Weir DR, et al. The desoxyribosenucleic acid (DNA) content in spermatozoa of fertile and infertile human males. Chromosoma 1953;6:61-78.

6. Ringertz NR, Gledhill BL, Darzynkiewicz Z. Changes in deoxyribonucleoprotein during spermiogenesis in the bull. Sensitivity of DNA to heat denaturation. Exp Cell Res 1970;62:204-18.

7. Agarwal A, Cho CL, Esteves SC. Should we evaluate and treat sperm DNA fragmentation? Curr Opin Obstet Gynecol 2016;28:164-71.

8. Agarwal A, Majzoub A, Esteves SC, et al. Clinical utility of sperm DNA fragmentation testing: practice recommendations based on clinical scenarios. Transl Androl Urol 2016;5:935-50.

9. Majzoub A, Agarwal A, Cho CL, et al. Sperm DNA fragmentation testing: a cross sectional survey on current practices of fertility specialists. Transl Androl Urol 2017;6:S710-9.

10. Esteves SC, Agarwal A, Cho CL, et al. A StrengthsWeaknesses-Opportunities-Threats (SWOT) analysis on the clinical utility of sperm DNA fragmentation testing in specific male infertility scenarios. Transl Androl Urol 2017;6:S734-60.

11. Cho CL, Agarwal A, Majzoub A, et al. Clinical utility of sperm DNA fragmentation testing: concise practice recommendations. Transl Androl Urol 2017;6:S366-73.

12. Agarwal A, Cho CL, Majzoub A, et al. The Society for Translational Medicine: clinical practice guidelines for sperm DNA fragmentation testing in male infertility. Transl Androl Urol 2017;6:S720-33.

13. Practice Committee of the American Society for Reproductive Medicine. Diagnostic evaluation of the infertile male: a committee opinion. Fertil Steril 2015;103:e18-25.

14. Jarow J, Sigman M, Kolettis PN, et al. The optimal evaluation of the infertile male: best practice statement reviewed and validity confirmed 2011. Available online: https://www.auanet.org/education/guidelines/maleinfertility-d.cfm 
15. Jungwirth A, Diemer T, Dohle GR, et al. Guidelines on male infertility. Available online: https://uroweb.org/ guideline/male-infertility/

16. Cohrs RJ, Martin T, Ghahramani P, et al. Translational

Cite this article as: Agarwal A, Cho CL, Majzoub A, Esteves SC. Frontiers in clinical andrology. Transl Androl Urol 2017;6(Suppl 4):S343-S345. doi: 10.21037/tau.2017.09.07 medicine definition by the European Society for Translational Medicine. New Horiz Transl Med 2015;2:86-8. 